\title{
Increased CD16 expression on NK cells is indicative of antibody-dependent cell-mediated cytotoxicity in chronic-active antibody-mediated rejection
}

\author{
Kasia A. Sablik ${ }^{*, 1}$, Nicolle H.R. Litjens ${ }^{1}$, Mariska Klepper, Michiel G.H. Betjes \\ Department of Nephrology and Transplantation, Erasmus Medical Center, Rotterdam, The Netherlands
}

\section{A R T I C L E I N F O}

\section{Keywords:}

Kidney transplantation

Biomarkers

Chronic active antibody mediated rejection

Circulating immune cells

\begin{abstract}
A B S T R A C T
Chronic-active antibody mediated rejection (c-aABMR) contributes significantly to late renal allograft failure. The antibodies directed against donor-derived antigens, e.g. anti-HLA antibodies, cause inflammation at the level of the microvascular endothelium. This is characterized by signs of local activation of the complement system and accumulation of immune cells within the capillaries. Non-invasive biomarkers of c-aABMR are currently not available but could be valuable for early detection. We therefore analyzed the activation profiles of circulating T and B cells, NK cells and monocytes in the peripheral blood of 25 kidney transplant recipients with c-aABMR and compared them to 25 matched recipients to evaluate whether they could serve as a potential biomarker.

No significant differences were found in the total percentage and distribution of NK cells, B cells and T cells between the c-aABMRpos and c-aABMRneg cases. There was however a higher percentage of monocytes present in c-aABMRpos cases $(p<.05)$. Additionally, differences were found in activation status of circulating monocytes, NK cells and $\gamma \delta$ T cells, mainly concerning the activation marker CD16. Although statistically significant, these differences were not sufficient for use as a biomarker of c-aABMR.
\end{abstract}

\section{Introduction}

Chronic-active antibody-mediated rejection (c-aABMR) is a significant long-term complication after kidney transplantation. It causes severe graft injury and c-aABMR is now recognized as one of the major barriers for long term renal allograft survival [1-4].

The histomorphological lesions of c-aABMR develop over time and are associated with recurrent and episodic endothelial activation caused by antibodies recognizing donor-specific antigens on the renal endothelial cells. The subsequent inflammation is specifically found at the level of the microcirculation [4-7]. Pre-transplant donor specific antibodies against HLA (DSA) and de novo DSA, which develop after transplantation, play an important role in the development of c-aABMR [8]. However, in a substantial number of cases DSA cannot be detected in the serum at time of diagnosis [9-12].

The antibodies on the endothelial cells are targeted by leukocytes bearing Fc- $\gamma$ receptors. In turn, the Fc-receptor-mediated activated cells can produce pro-inflammatory cytokines such as interferon-gamma (IFN- $\gamma$ ) and upregulated expression of levels IFN- $\gamma$ in the graft and serum have been described during ABMR $[13,14]$. In combination with a variable degree of local complement activation, the activation of immune cells results in endothelial injury. The persistent endothelial injury leads to structural histomorphological changes of the glomeruli with loss of fenestration and duplication/multilamination of the basement membranes; more specifically known as transplant glomerulopathy (TG) [6,15-18].

All circulating immune cells bearing Fc-receptors can potentially be activated by.

endothelial cells in c-aABMR, however, in particular innate immune cells such as monocytes and NK cells are typically present in the glomerular and peritubular capillaries [14,19].

In addition, specific $\mathrm{T}$ cell subsets have been recognized as potentially important immune modulators in rejection. For instance, cytomegalovirus-responsive (CMV) $\gamma \delta \mathrm{T}$ cells are possible effector cells in antibody mediated rejection and Baeten et al., identified increased numbers of CD8 + CD28 - effector lymphocytes in patients with chronic graft rejection [20,21].

Non-invasive biomarkers of c-aABMR are currently not available but could be valuable for early detection and subsequent treatment. Whether the continuous activation of immune cells can be detected in

*Corresponding author at: Erasmus Medical Center, Department of Nephrology \& Transplantation, room NA2105, P.O. Box 2040, 3000 CA Rotterdam, The Netherlands.

E-mail address: k.sablik@erasmusmc.nl (K.A. Sablik).

${ }^{1}$ These authors contributed equally to this manuscript. 
Table 1

Clinical and demographic characteristics (at time of for cause biopsy).

\begin{tabular}{|c|c|c|c|c|}
\hline & Total (50) & c-aABMRpos cases $(n=25)$ & c-aABMRneg cases $(\mathrm{n}=25)$ & p-Value \\
\hline Women, n (\%) & $15(30)$ & $8(32)$ & $7(28)$ & 0.76 \\
\hline Age of patient, yr, median (IQR) & $52(39-66)$ & $53(44-66)$ & $51(35-68)$ & 0.85 \\
\hline Living donor, $\mathrm{n}(\%)$ & $41(82)$ & $21(84)$ & $20(80)$ & 0.71 \\
\hline Prior kidney transplant, n (\%) & $10(20)$ & $6(24)$ & $4(16)$ & 0.48 \\
\hline Donor age, yr, median (IQR) & $51(43-61)$ & $52(46-62)$ & $48(40-48)$ & 0.21 \\
\hline PRA current, median (IQR) & $0(0-5)$ & $2(0-8)$ & $0(0-0)$ & 0.17 \\
\hline Donor Specific Antibodies, n (\%) & - & $12(48)$ & - & \\
\hline HLA mismatch, median (IQR) & $3(2-4)$ & $3(2-5)$ & $3(1-4)$ & 0.85 \\
\hline \multicolumn{5}{|l|}{ Maintenance immunosuppression, n (\%) } \\
\hline Tacrolimus/cyclosporine & $47(94)$ & $23(92)$ & $24(96)$ & 0.55 \\
\hline mTOR inhibitor & $1(2)$ & $0(0)$ & $1(4)$ & 0.31 \\
\hline Steroids & $24(48)$ & $11(44)$ & $13(52)$ & 0.57 \\
\hline Mycophenolate mofetil & $43(86)$ & $24(96)$ & $19(76)$ & 0.04 \\
\hline Other & $3(6)$ & $0(0)$ & $3(12)$ & 0.08 \\
\hline \multicolumn{5}{|l|}{ Maintenance immunosuppression, $\mathrm{n}(\%)$} \\
\hline Triple immunosuppression & $18(36)$ & $8(32)$ & $10(40)$ & 0.56 \\
\hline Double immunosuppression & $32(64)$ & $17(68)$ & $15(60)$ & \\
\hline \multicolumn{5}{|l|}{ Primary kidney disease, $\mathrm{n}(\%)$} \\
\hline Diabetic nephropathy & $9(18)$ & $5(20)$ & $4(16)$ & 0.36 \\
\hline Hypertensive nephropathy & $5(10)$ & $1(4)$ & $4(16)$ & \\
\hline Polycystic kidney disease & $7(14)$ & $5(20)$ & $2(8)$ & \\
\hline Primary glomerulopathy & $13(26)$ & $5(20)$ & $8(32)$ & \\
\hline Other & $15(30)$ & $9(36)$ & $6(24)$ & \\
\hline Unknown & $1(2)$ & $0(0)$ & $1(4)$ & \\
\hline Time to for cause biopsy, yr, median (IQR) & $4.2(3.0-10.6)$ & $4.2(2.9-11.6)$ & $4.1(3.0-11.0)$ & 0.92 \\
\hline Graft function (ml/min), median (IQR) & $33(28-44)$ & $33(27-43)$ & $36(29-49)$ & 0.55 \\
\hline
\end{tabular}

the circulation of patients with c-aABMR and may serve as a potential biomarker, is unknown. In this study we investigated monocytes, NK cells, B cells and $\mathrm{T}$ cells with their subsets in combination with a variety of activation markers expressed on these cells (HLA-DR, CD25, CD38 and CD71) in relation to c-aABMR.

\section{Patients and methods}

\subsection{Study population}

This case-control study included 25 cases of kidney transplant recipients diagnosed with chronic active antibody-mediated rejection (caABMRpos) and 25 matched controls (c-aABMRneg). Blood samples were collected at time of diagnosis between June 2016 and November 2017 at the Erasmus University Medical Center (Rotterdam, The Netherlands).

The c-aABMRneg cases were matched for age, gender and time after transplantation and had a for-cause renal biopsy which showed no evidence of rejection. All renal biopsies were for-cause and evaluated by an experienced renal pathologist based on the then current Banff classification [22,23].

Kidney transplant recipients gave written informed consent and the study was approved by the Medical Ethical Committee of the Erasmus MC (MEC-2017-115). The study was conducted in accordance with the Declaration of Helsinki and the Declaration of Istanbul.

\subsection{Data collection and isolation of serum and peripheral blood mononuclear cells (PBMCs)}

Demographic and baseline transplantation characteristics were collected for all patients at time of for-cause biopsy. Blood was drawn from the kidney transplant recipients prior to the for-cause biopsy. Peripheral blood mononuclear cells (PBMCs) were isolated from heparinized blood samples by using Ficoll-Paque Plus (GE healthcare, Uppsala, Sweden) [24]. The isolated PBMCs were washed, frozen at $10 \times 10^{6}$ /vial in RPMI-1640 with Glutamax (GibcoBRL) supplemented with $100 \mathrm{IU} / \mathrm{mL}$ penicillin/streptomycin and $10 \%$ heat-inactivated pooled human serum and 10\% dimethyl sulphoxide (Sigma Aldrich, Darmstadt, Germany) in liquid nitrogen until further use. One vial of PBMCs was thawed in RPMI-1640 (Gibco BRL) containing penicillin/ streptomycin and DNase (Sigma Aldrich), washed, the cells counted and viability assessed using trypan blue.

Serum was collected upon centrifuging the coagulation tube for $10 \mathrm{~min}$ at $3000 \mathrm{rpm}$ and stored at $1 \mathrm{~mL} /$ ampoule at $-80{ }^{\circ} \mathrm{C}$ for determination of DSA.

\subsection{Characterization PBMCs by flow cytometry}

PBMCs were characterized for proportions of different populations as well as their activation state using flow cytometry. The activation state was evaluated by analyzing frequencies and median fluorescence intensity of human leukocyte antigen-DR (HLA-DR)-, CD25-, CD38- or CD71-expressing cells. HLA-DR is expressed on B cells, monocytes, activated T cells and activated NK cells. CD25 is the $\alpha$-chain of the IL-2 receptor and its expression is also increased upon activation of $\mathrm{T}$ cells and B cells. CD38 is a glycoprotein involved in cell adhesion, signal transduction and calcium signaling and expressed on T cells, B cells, monocytes and NK cells. CD71 is the transferrin receptor involved in iron transport from transferrin into cells by endocytosis and its expression is increased upon activation on $\mathrm{T}$ and $\mathrm{B}$ cells.

PBMCs were divided over 5 different polystyrene FACS tubes (Becton Dickinson, BD; Erembodegem, Belgium) and stained using different antibody panels. Below a brief description of the panels is given. Detailed information with respect to the different antibodies within each panel is provided within Supplemental Table 1.

In panel 1, percentages of monocyte subsets and NK cells are determined as well as their activation state. Using the combination of $\mathrm{CD} 14$ and $\mathrm{CD} 16$, classical $\left(\mathrm{CD} 14^{++} \mathrm{CD} 16^{-}\right)$, intermediate $\left(\mathrm{CD} 14^{+} \mathrm{CD} 16^{+}\right)$and non-classical $\left(\mathrm{CD} 14^{+} \mathrm{CD} 16^{++}\right)$monocytes were identified within PBMCs as described by Ziegler-Heitbrock et al. [25]. The NK cells are identified as negative for CD3 and CD19 and positive for CD56 either co-expressing CD16 or not [26].

In panel 2, percentages of $\mathrm{B}$ cell subsets are determined as described by Kaminski et al. as well as their activation state [27]. B cells are 

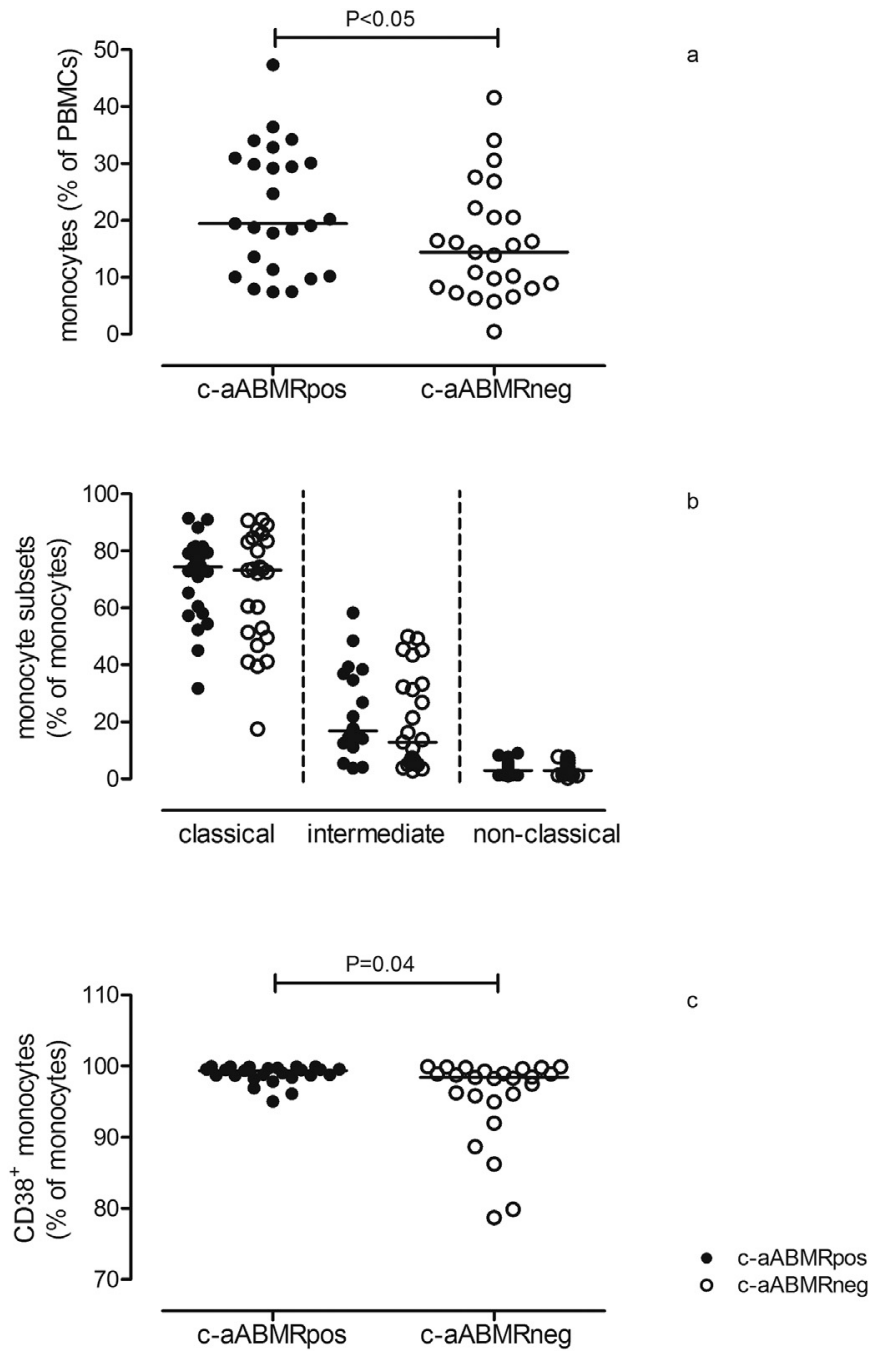

Fig. 1. a. Total percentage of monocytes in c-aABMRpos and c-aABMRneg cases; b. Distribution of monocyte subsets; c. CD38 expression on monocytes.

identified as $\mathrm{CD} 19^{+}$and using the combination of IgD and CD27, naïve B cells $\left(\mathrm{CD}^{2} 7^{-} \mathrm{IgD}^{+}\right)$can be dissected from non-class switched $\left(\mathrm{CD}_{27}{ }^{+} \mathrm{IgD}^{+}\right)$, class-switched $\left(\mathrm{CD} 27^{+} \mathrm{IgD}^{-}\right)$and double negative (DN, $\mathrm{CD}^{2} 7^{-} \mathrm{IgD}^{-}$) memory B cells. Plasma blast can be identified within the circulation by combining expression of $\mathrm{CD} 27$ and $\mathrm{CD} 38$ $\left(\mathrm{CD} 27^{++} \mathrm{CD} 38^{++}\right)$and transitional B cells by high expression of both CD24 as well as CD38.

In panel 3 we evaluate percentages of T cells expressing V $\delta 1$ and V82 T cell receptor (TCR) as well as their activation state. As these cells have been implicated in antibody-mediated rejection in kidney transplant recipients and also can express CD16, we also determined percentages and median fluorescence intensity (MFI) of CD16 (Fc gamma III receptor) [21]. Gamma delta $\mathrm{T}$ cells are identified as $\mathrm{CD}^{+}$and positive for either $\mathrm{V} \delta 1$ or $\mathrm{V} \delta 2$. Within the circulation predominantly $\mathrm{V} \delta 2^{+} \mathrm{V} \delta 1 \gamma \delta^{-} \mathrm{T}$ cells are observed [28].

In panel 4 , we measure frequencies of $\mathrm{CD}^{+}$and $\mathrm{CD}^{+} \mathrm{T}$ cells (expressing the alpha beta TCR) as well as their activation state and in panel 5 , we characterize the different $\mathrm{CD}^{+}{ }^{+}$and $\mathrm{CD} 8^{+} \mathrm{T}$ cell subsets in more detail by evaluating their differentiation status. The differentiation status was based on the study by Sallusto et al., as described in detail previously by Betjes et al. [29,30]. Briefly, expression of CD45RO (marker for memory T cells) and CCR7 (a chemokine receptor which facilitates $\mathrm{T}$ cells to home to secondary lymphoid organs) was used to determine naive $\left(\mathrm{CD}_{45 \mathrm{RO}^{-}} \mathrm{CCR7}^{+}\right)$and different memory T-cell populations. Different memory T-cell subsets were defined as central memory (CM) T cells $\left(\mathrm{CD} 45 \mathrm{RO}^{+} \mathrm{CCR}^{+}{ }^{+} \mathrm{T}\right.$ cells), effector memory (EM) $\mathrm{T}$ cells $\left(\mathrm{CD} 45 \mathrm{RO}^{+} \mathrm{CCR7}^{-} \mathrm{T}\right.$ cells) and EMRA $\mathrm{T}$ cells (highly differentiated $\mathrm{CD}^{4} 5 \mathrm{RA}^{+} \mathrm{CCR7}^{-}$effector memory T cells). Furthermore, we have determined frequencies of more differentiated $\mathrm{T}$ cells based on the loss of the co-stimulatory molecule CD28 on the cell surface of these cells.

Upon staining the cells for $30 \mathrm{~min}$ at room temperature with the different antibody cocktails, the samples were washed using BD FACSflow (BD), measured on the FACSCanto II (BD; 3 laser, 8 color configuration 4:2:2) and analyzed using Kaluza software version 1.3 (Beckman Coulter BV., Woerden, The Netherlands) generating a linear value for median fluorescence intensity of the different activation markers. Multiplying this value by 256 makes it comparable to those obtained by other flow cytometry analysis software. To be able to dissect the different populations of cells and analyze their activation state, we aimed to acquire at least 50.000 PBMCs. A typical example of flow cytometric analyses and gating strategy for the different cells is given in Supplemental Fig. 1A-D and in Supplemental Fig. 2A-F the gating of the different activation markers for particular cell population is given. Fluorescence minus one (FMO) controls were used to determine positivity for each activation marker. Data of all activation markers are depicted in Supplemental Tables 2-6.

\subsection{Statistical analysis}

Normally distributed data are expressed as mean $+/-\mathrm{SD}$, nonnormally distributed data as median (IQR). The total cell populations and their distribution were expressed as median percentages (IQR). The activation state was evaluated by analyzing frequencies of cells positive for an activation marker and the median fluorescence intensity (MFI) of the positive fraction as a marker for expression level of the activation marker. All frequencies are expressed as median percentages (IQR) and the MFI's as median values (IQR).

The statistical analyses were performed using Graphpad Prism 6 and SPSS software version 24. Statistical significance was calculated by Fisher's exact test, Mann-Whitney- $U$ test and two-way ANOVA. A $p$ value of $<0.05$ was considered statistically significant.

\section{Results}

\subsection{Baseline characteristics}

A detailed description of demographics and clinical characteristics of the study population is given in Table 1 . The majority of patients were male $(70 \%)$ with a median age of 52 and transplanted with a kidney from a living donor (82\%). Most patients received tacrolimus and mycophenolate mofetil $(94 \%$ and $86 \%)$ as their immunosuppressive therapy. The biopsies were taken at a median of 4.2 years after transplantation (c-aABMRpos 4.2 yrs. versus caABMRneg 4.1 yrs.; $p=.92$ ). The majority of c-aABMRneg cases had signs of chronic damage attributed to CNI toxicity in their biopsy.

The CMV serostatus of the patients is of interest as CMV primo infection and latency are associated with increased numbers of differentiated $\mathrm{CD}^{+}$and $\mathrm{CD}^{+}{ }^{+} \mathrm{T}$ cells [31]. However, no differences were found in CMV mismatch $(p=.72)$. A total of 10 patients had a CMV mismatch in which the donor was CMV positive and the acceptor CMV negative ( $n=6$ for cases, $n=4$ for controls). Of those 10 mismatch patients, three cases and two controls had undergone seroconversion after transplantation.

\subsection{Monocytes and NK cells}

The total percentage of monocytes was significantly higher in the caABMRpos cases $(19.5 \% ; 10.8-30.5 \%)$ compared to the c-aABMRneg cases $(14.4 \%$; $8.2-21.4 \%)(p<.05)$ even though the distribution of classical $\left(\mathrm{CD} 14^{++} \mathrm{CD} 16^{-}\right)$, intermediate $\left(\mathrm{CD} 14^{+} \mathrm{CD} 16^{+}\right)$and non- 

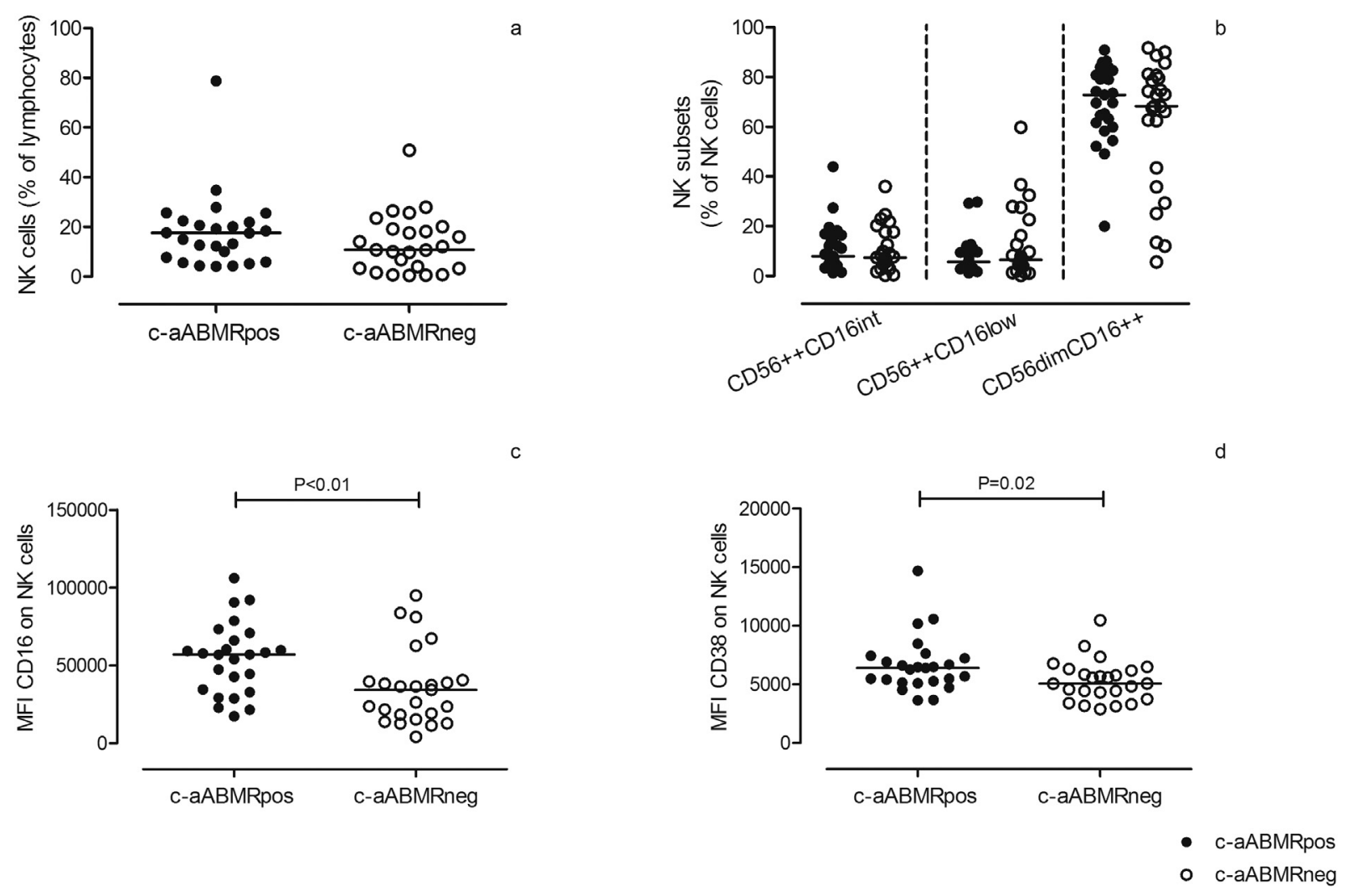

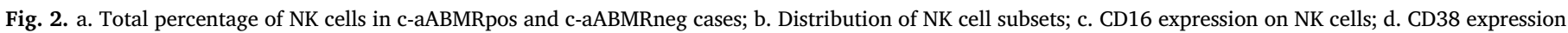
on NK cells.

classical $\left(\mathrm{CD} 14^{+} \mathrm{CD} 16^{++}\right)$monocytes was similar. However, the caABMRpos cases showed a significantly higher percentage of monocytes expressing the activation marker CD38 compared to the caABMRneg cases (c-aABMRpos 99.3\% versus c-aABMRneg 98.4\%; $p=.04$ ) (Fig. 1a-c). Upon analysis DSA presence in the c-aABMRpos cases was not associated with the significant differences.

Similar to the data on monocytes, the percentage of NK cells in caABMR cases tended to be higher $(17.5 \% ; 6.8-22.1 \%)$ compared to the c-aABMRneg cases $(10.7 \%$; 3.4-19.6\%) although statistical significance was not reached $(p=.14)$ and NK cell subsets distribution was similar $(p=.51)$. However, NK cells of c-aABMRpos cases showed a significantly higher expression of CD16 (Fc $\gamma$ III receptor) and CD38 $(p<.01$ and $p=.02)$ based on MFI. The median CD16 MFI of NK cells of c-aABMRpos cases was 56,965 $(33734-68,403)$ and for NK cells of caABMRneg cases 34,345 (16878-40,092). For CD38 MFI these values were 6403 (5206-7336) and 5079 (4040-6240), respectively (Fig. 2a-d). The significant changes found in the expression profile of NK cells showed no association with DSA presence in the c-aABMRpos cases.

\subsection{B cells}

The overall percentage of B cells was similar for c-aABMRpos cases and c-aABMRneg cases $(p=.68$; c-aABMRpos $2.7 \%(1.4-7.0 \%)$; caABMRneg $4.2 \%(1.6-5.5 \%)$ ), as was the distribution of B cell subsets $(p=.95)$. Of interest was the tendency for higher expression of the activation marker HLA-DR on B cells in patients with c-aABMR $(p=.09)$ (Fig. 3a-c). The median MFI of HLA-DR for B cells of caABMRpos cases was 2202 (1967-2728) and for c-aABMRneg cases 2132 (1750-2698).

\subsection{T cells}

The total percentage of $\mathrm{T}$ cells $\left(\mathrm{CD}^{+}\right.$cells) was $69.9 \%$ (58.5-79.4\%) for the c-aABMRpos cases and 68.1\% (55.7-80.3\%) for the c-aABMRneg cases which did not differ significantly $(p=.65)$. Neither was there a difference in frequencies of $\mathrm{CD}^{+}(p=.79)$ and $\mathrm{CD}^{+} \mathrm{T}$ cells $(p=.77)$ (Fig. 4a-b). Furthermore, no significant difference in expression of activation markers (HLA-DR, CD38, CD71 and CD25) were found for $\mathrm{CD}^{+}, \mathrm{CD}^{+}$and $\mathrm{CD} 8^{+} \mathrm{T}$ cells.

Additionally, we analyzed in detail the differentiation status of the $\mathrm{CD}^{+}$and $\mathrm{CD} 8^{+} \mathrm{T}$ cell subsets. However, similar percentages were found for naïve, CM, EM, EMRA and CD28null subsets within CD4+ and $\mathrm{CD} 8+\mathrm{T}$ cells in c-aABMRpos and c-aABMRneg cases (Fig. 4c-d).

\section{5. $\gamma \delta$ T cells}

The frequency of $\gamma \delta$ T cells did not differ significantly between caABMRpos and c-aABMRneg cases $(p=.88$; c-aABMRpos $2.4 \%$ (1.3-4.7\%); c-aABMRneg $2.2 \%(1.5-5.8 \%)$ ). The ratio of $\mathrm{V} \delta 1$ and $\mathrm{V} \delta 2$ subsets also did not differ significantly when comparing cases to controls $(p=.40)$ (Fig. 5a-b). Upon evaluation of the activation markers, the expression of HLA-DR was significantly higher in the c-aABMRneg cases $(p=.02$; MFI c-aABMRpos 637 (590-708); MFI c-aABMRneg 712 (641-824)), as well as the expression of CD16 ( $\mathrm{p}=.02$; MFI $\mathrm{c}$ aABMRpos 837 (684-1280); MFI c-aABMRneg 1277 (955-2381)) (Fig. 5c-d).

\section{Discussion}

Chronic active antibody mediated rejection is an important cause of late renal allograft failure [1,2]. With little knowledge on prevention, the underlying pathomechanisms as well as the absence of an effective treatment, c-aABMR remains a clinical challenge [32]. Unfortunately, 

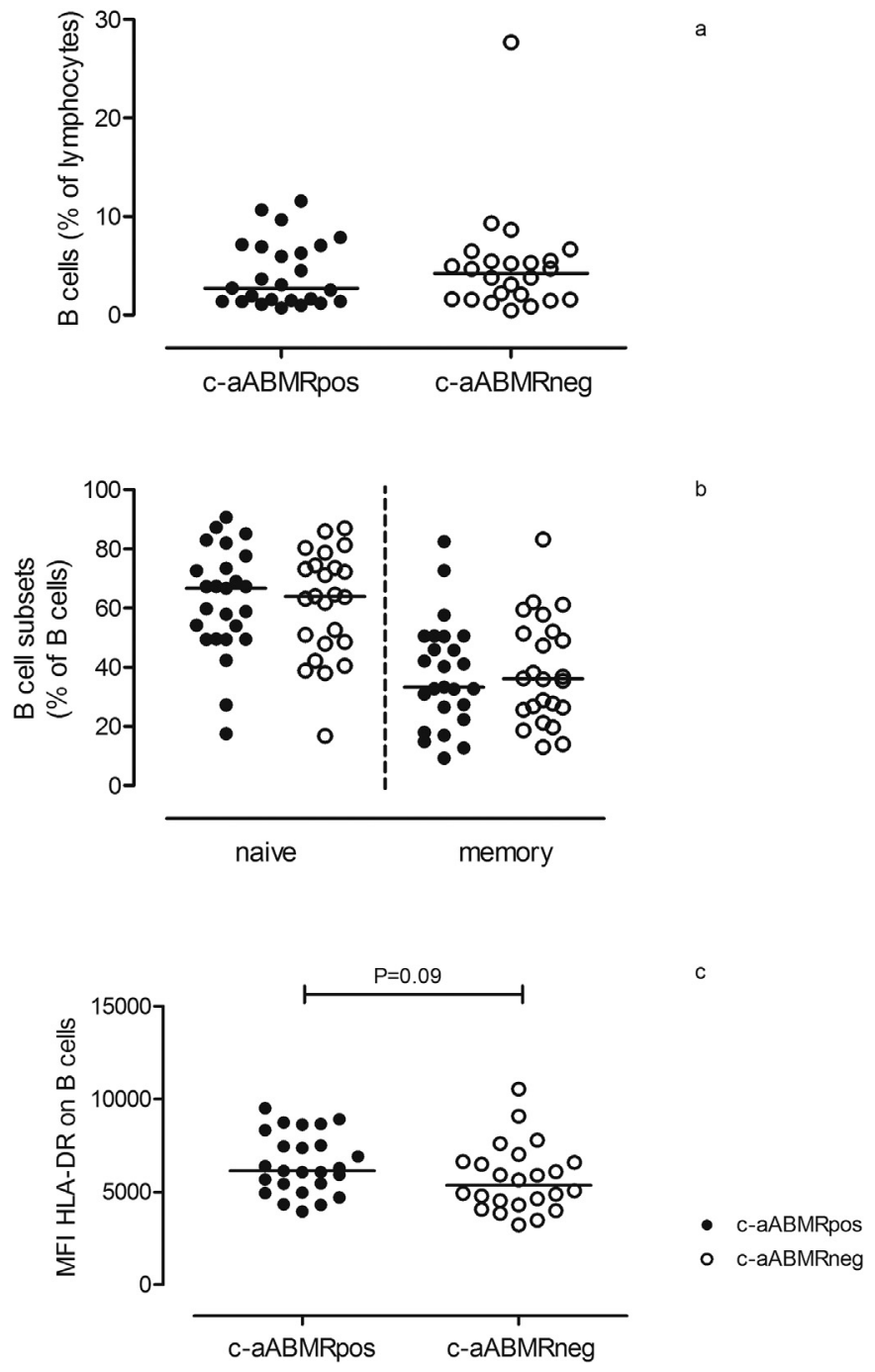

Fig. 3. a. Total percentage of B cells in c-aABMRpos and c-aABMRneg cases; b. Distribution of B cell subsets; c. HLA-DR expression on B cells.

until present, the gold standard for the diagnosis of c-aABMR remains renal biopsy due to the absence of adequate biomarkers. In this study we provide the first in-depth analysis into the different leukocyte subsets and their activation markers in the peripheral blood of patients with c-aABMR in comparison to matched controls.

We found similar distribution in c-aABMRpos cases and caABMRneg cases for NK cells, B cells, T cells and their specific subsets. However, subtle differences were present in the overall percentage of monocytes and expression of activation markers with generally a more activated profile of circulating monocytes and NK cells in patients with c-aABMR.

Monocyte subset distribution has been implicated as a potential biomarker for acute rejection in renal transplant recipients [19], but no data are present on the relation with development of either acute or chronic ABMR. The results of this study did not find differences in frequency of classical, intermediate and non-classical monocytes but did show a higher overall percentage of monocytes in patients with caABMR. CD38 expression was significantly higher in cases of c-aABMR but this seemed to be related to several unexplained outliers in the caABMRneg group and MFI of the CD38 expression was comparable in both groups.

Similar to the monocytes, the median percentage of NK cells tended to be higher in the c-aABMR cases with increased expression of the activation markers CD16 and CD38. It has been hypothesized by several groups that effector mechanisms other than complement can be activated through DSA [33,34]. An ABMR model was proposed in which the contribution of NK cells at endothelial level is mediated by CD16 engagement to DSA [35]. Damage is inflicted through antibody-dependent cell-mediated cytotoxicity (ADCC) mediated by Fc receptors on the cell surface that bind IgG antibodies (CD16) [14,21,36]. The interaction through the low affinity FcR type III (CD16) is crucial as it stimulates regulation of proliferation, migration of other leukocytes and endothelial cytotoxicity by releasing granule content and pro-inflammatory CD16-inducible cytokines. The increased expression of CD16 would confirm this possible underlying pathophysiological mechanism leading to c-aABMR $[13,35,37,38]$.

Activated T cells may also be a source of IFN- $\gamma$ production in caABMR but we found no differences in the percentage of circulating $\mathrm{T}$ cells, differentiation and activation status. Therefore, in line with previously published models our data are not in support of a major pathophysiological role for T cells in c-aABMR $[13,39,40]$.

Of interest in this respect is the observation that a specific subset of $\mathrm{T}$ cells namely $\mathrm{V} \delta 2^{\text {neg }} \gamma \delta \mathrm{T}$ cells also express CD16 and their ADCC function is triggered after interaction with cell bound antibodies. These cells are suspected to play a role in acute ABMR as $\gamma \delta$ T cells were found in peritubular capillaries during ABMR and their peripheral blood expansion may be considered a poor prognostic factor for allograft function [21]. Of interest, the number of these cells in the peripheral blood is related to the CMV serostatus as CMV infection causes activation and expansion of $\mathrm{CD} 16^{+} \gamma \delta$ T cells.

However, the data from this study do not show significant differences between the number and subset distribution of $\gamma \delta$ T cells between c-aABMRpos and c-aABMRneg cases. In addition, no difference with respect to frequency of CMV-seropositive kidney transplant recipients was observed between the two groups Remarkable was the significantly lower expression of CD16 and HLA-DR in the c-aABMRneg cases. As previously postulated by van den Bosch et al., it is possible that these cells have migrated to the inflamed graft rather than remained in the circulation [19].

Furthermore, we found no differences in the percentage of B cells, their differentiation status and activation markers. These findings are similar to those of Hidalgo et al. who established a DSA selective transcript algorithm and found no B cell transcripts in renal biopsies with ABMR [14]. Previously, it was demonstrated that the expression of B cell associated transcripts (BAT) are features of scarring and injury. BAT are associated with time post-transplantation and have no relation to ABMR or DSA [41].

Until present, the majority of studies have focused on the histological characterization and transcriptomes of specific cells in early rejection. Although there are several studies that have analyzed peripheral blood in search of potential predictive markers, most have investigated pre-transplant samples rather than samples at time of rejection $[19,42,43]$. This is the first study to, in detail, describe the circulating immune cells and their activation and differentiation markers at time of c-aABMR diagnosis. In spite of the limited numbers of included patients, this study contains a clearly defined group of caABMR patients with a time-, age- and gender-matched control group.

\subsection{Conclusion}

In conclusion, our current results demonstrate differences in the numbers and activation status of circulating monocytes, NK cells and $\gamma \delta$ $\mathrm{T}$ cells in the peripheral blood of c-aABMRpos and c-aABMRneg patients. Taken together, the data are consistent with activation of cells that bear the Fc receptor CD16 and fit within the hypothesis that interaction with antibodies on renal endothelial cells leave a footprint in the circulating CD16pos immune cell populations. However, these are subtle differences and are not fit to serve as biomarkers. Nevertheless, the data are of interest and indicate an important role for CD16 positive innate immune cells in the pathogenesis of c-aABMR. 

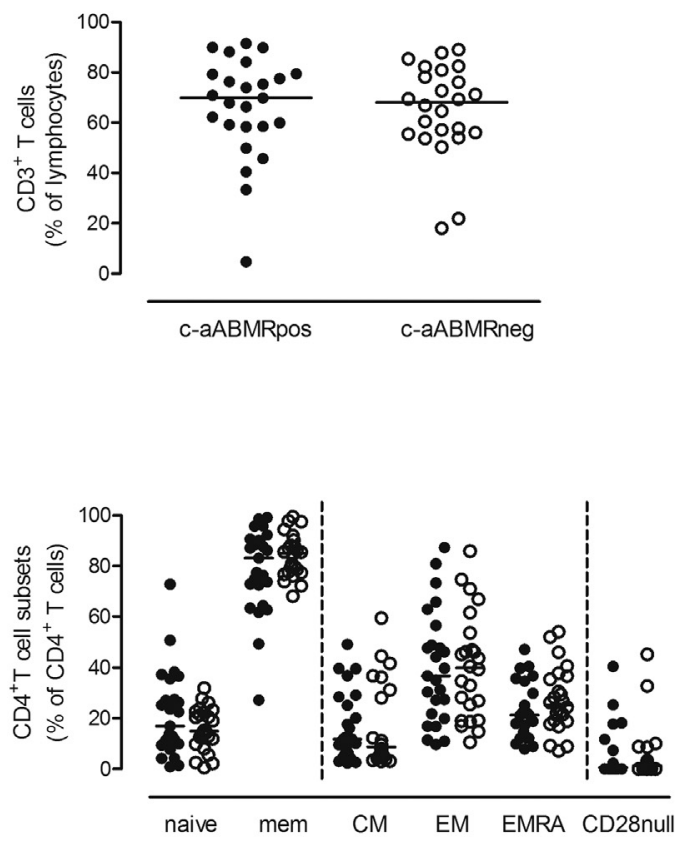

a

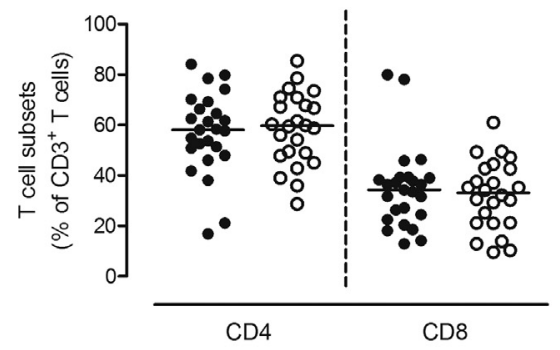

c

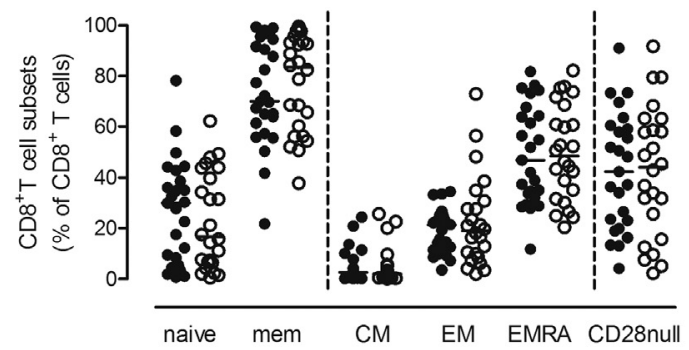

b

d

- c-aABMRpos

o c-aABMRneg

Fig. 4. a. Total percentage of $\mathrm{CD}^{+}{ }^{+} \mathrm{T}$ cells in c-aABMRpos and c-aABMRneg cases; b. Distribution of $\mathrm{CD} 4^{+}$and $\mathrm{CD} 8^{+} \mathrm{T}$ cells; c. Distribution of CD4 ${ }^{+} \mathrm{T}$ cell subsets; d. Distribution of $\mathrm{CD}^{+} \mathrm{T}$ cell subsets.
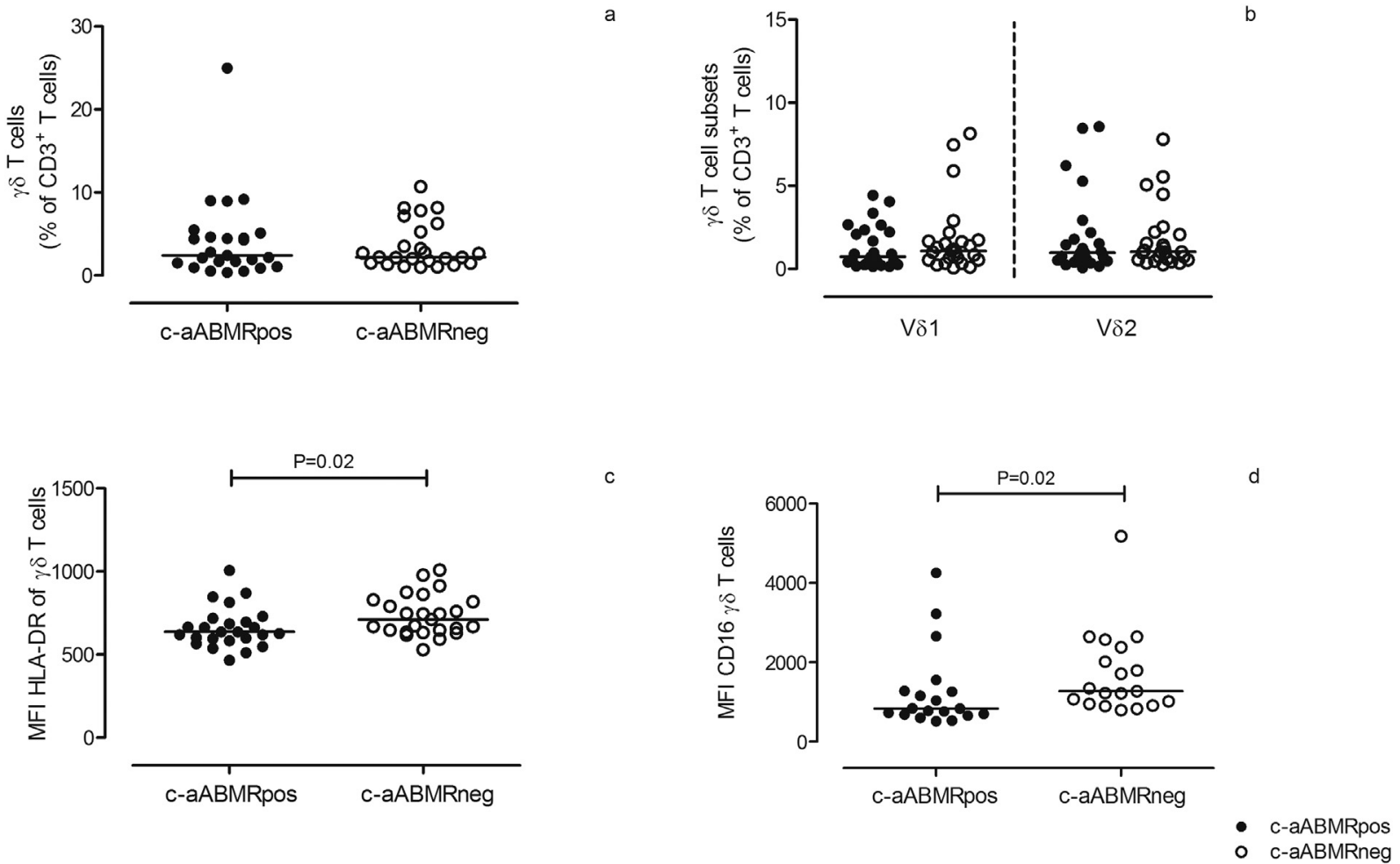

Fig. 5. a. Total percentage of $\gamma \delta$ T cells in c-aABMRpos and c-aABMRneg cases b. Distribution of $\gamma \delta$ T cell subsets in CD3 ${ }^{+}$T cells; c. HLA-DR expression on $\gamma \delta$ T cells; d. CD16 expression on $\gamma \delta$ T cells. 
Supplementary data to this article can be found online at https:// doi.org/10.1016/j.trim.2019.02.005.

\section{Declarations of interest}

None.

\section{CRediT authorship contribution statement}

Kasia A. Sablik: Conceptualization, Data curation, Formal analysis, Methodology, Visualization, Writing - original draft, Writing - review \& editing. Nicolle H.R. Litjens: Conceptualization, Data curation, Formal analysis, Methodology, Resources, Visualization, Writing - original draft, Writing - review \& editing. Mariska Klepper: Data curation, Formal analysis, Resources, Validation, Writing - review \& editing. Michiel G.H. Betjes: Conceptualization, Methodology, Supervision, Validation, Visualization, Writing - original draft, Writing - review \& editing.

\section{Acknowledgements}

This research did not receive any specific grant from funding agencies in the public, commercial, or not-for-profit sectors.

\section{References}

[1] K.E. Lamb, S. Lodhi, H.U. Meier-Kriesche, Long-term renal allograft survival in the United States: a critical reappraisal, Am. J. Transplant. 11 (3) (2011) 450-462.

[2] R.S. Gaston, J.M. Cecka, B.L. Kasiske, et al., Evidence for antibody-mediated injury as a major determinant of late kidney allograft failure, Transplantation. 90 (1) (2010) 68-74.

[3] J. Sellares, D.G. de Freitas, M. Mengel, et al., Understanding the causes of kidney transplant failure: the dominant role of antibody-mediated rejection and nonadherence, Am. J. Transplant. 12 (2) (2012) 388-399.

[4] G. Einecke, B. Sis, J. Reeve, et al., Antibody-mediated microcirculation injury is the major cause of late kidney transplant failure, Am. J. Transplant. 9 (11) (2009) 2520-2531.

[5] F.G. Cosio, J.M. Gloor, S. Sethi, M.D. Stegall, Transplant glomerulopathy, Am. J. Transplant. 8 (3) (2008) 492-496.

[6] J.M. Gloor, S. Sethi, M.D. Stegall, et al., Transplant glomerulopathy: subclinical incidence and association with alloantibody, Am. J. Transplant. 7 (9) (2007) 2124-2132.

[7] B. Sis, G.S. Jhangri, J. Riopel, et al., A new diagnostic algorithm for antibodymediated microcirculation inflammation in kidney transplants, Am. J. Transplant. 12 (5) (2012) 1168-1179.

[8] B. Sis, P.M. Campbell, T. Mueller, et al., Transplant glomerulopathy, late antibodymediated rejection and the ABCD tetrad in kidney allograft biopsies for cause, Am. J. Transplant. 7 (7) (2007) 1743-1752.

[9] W. Hanf, C.S. Bonder, P.T. Coates, Transplant glomerulopathy: the interaction of HLA antibodies and endothelium, J Immunol Res 2014 (2014) 549315.

[10] K.A. Sablik, M.C. Clahsen-van Groningen, Looman CWN, et al., Chronic-active antibody-mediated rejection with or without donor-specific antibodies has similar histomorphology and clinical outcome - a retrospective study, Transpl. Int. 31 (8) (2018) 900-908.

[11] P.F. Halloran, M. Merino Lopez, Pereira A. Barreto, Identifying subphenotypes of antibody-mediated rejection in kidney transplants, Am. J. Transplant. 16 (3) (2016) 908-920.

[12] E. Akalin, R. Dinavahi, S. Dikman, et al., Transplant glomerulopathy may occur in the absence of donor-specific antibody and C4d staining, Clin. J. Am. Soc. Nephrol. 2 (6) (2007) 1261-1267.

[13] M.D. Parkes, P.F. Halloran, L.G. Hidalgo, Mechanistic sharing between NK cells in ABMR and effector T cells in TCMR, Am. J. Transplant. 18 (1) (2018) 63-73.

[14] L.G. Hidalgo, B. Sis, J. Sellares, et al., NK cell transcripts and NK cells in kidney biopsies from patients with donor-specific antibodies: evidence for NK cell involvement in antibody-mediated rejection, Am. J. Transplant. 10 (8) (2010) 1812-1822.

[15] X. Zhang, E.F. Reed, Effect of antibodies on endothelium, Am. J. Transplant. 9 (11) (2009) 2459-2465.

[16] J. Fotheringham, C.A. Angel, W. McKane, Transplant glomerulopathy: morphology, associations and mechanism, Nephron Clin. Pract. 113 (1) (2009) (c1-7; discussion c7).
[17] M. Haas, Transplant glomerulopathy: the view from the other side of the basement membrane, J. Am. Soc. Nephrol. 26 (6) (2015) 1235-1237.

[18] L.C. Racusen, R.B. Colvin, K. Solez, et al., Antibody-mediated rejection criteria - an addition to the Banff 97 classification of renal allograft rejection, Am. J. Transplant. 3 (6) (2003) 708-714.

[19] T.P.P. van den Bosch, L.B. Hilbrands, R. Kraaijeveld, et al., Pretransplant numbers of $\mathrm{CD16}(+)$ monocytes as a novel biomarker to predict acute rejection after kidney transplantation: a pilot study, Am. J. Transplant. 17 (10) (2017) 2659-2667.

[20] D. Baeten, S. Louis, C. Braud, et al., Phenotypically and functionally distinct CD8+ lymphocyte populations in long-term drug-free tolerance and chronic rejection in human kidney graft recipients, J. Am. Soc. Nephrol. 17 (1) (2006) 294-304.

[21] T. Bachelet, L. Couzi, V. Pitard, et al., Cytomegalovirus-responsive gammadelta T cells: novel effector cells in antibody-mediated kidney allograft microcirculation lesions, J. Am. Soc. Nephrol. 25 (11) (2014) 2471-2482.

[22] A. Loupy, M. Haas, K. Solez, et al., The Banff 2015 kidney meeting report: current challenges in rejection classification and prospects for adopting molecular pathology, Am. J. Transplant. 17 (1) (2017) 28-41.

[23] M. Haas, A. Loupy, C. Lefaucheur, et al., The Banff 2017 kidney meeting report: revised diagnostic criteria for chronic active $\mathrm{T}$ cell-mediated rejection, antibodymediated rejection, and prospects for integrative endpoints for next-generation clinical trials, Am. J. Transplant. 18 (2) (2018) 293-307.

[24] N.H. Litjens, M. Huisman, C.C. Baan, C.J. van Druningen, M.G. Betjes, Hepatitis B vaccine-specific $\mathrm{CD} 4(+) \mathrm{T}$ cells can be detected and characterised at the single cell level: limited usefulness of dendritic cells as signal enhancers, J. Immunol. Methods $330(1-2)(2008) 1-11$.

[25] L. Ziegler-Heitbrock, T.P. Hofer, Toward a refined definition of monocyte subsets, Front. Immunol. 4 (2013) 23.

[26] J.A. Slyker, B. Lohman-Payne, G.C. John-Stewart, et al., The impact of HIV-1 infection and exposure on natural killer (NK) cell phenotype in Kenyan infants during the first year of life, Front. Immunol. 3 (2012) 399.

[27] D.A. Kaminski, C. Wei, Y. Qian, A.F. Rosenberg, I. Sanz, Advances in human B cell phenotypic profiling, Front. Immunol. 3 (2012) 302.

[28] I. Puig-Pey, F. Bohne, C. Benitez, et al., Characterization of gammadelta T cell subsets in organ transplantation, Transpl. Int. 23 (10) (2010) 1045-1055.

[29] F. Sallusto, D. Lenig, R. Forster, M. Lipp, A. Lanzavecchia, Two subsets of memory T lymphocytes with distinct homing potentials and effector functions, Nature. 401 (6754) (1999) 708-712.

[30] M.G. Betjes, A.W. Langerak, A. van der Spek, E.A. de Wit, N.H. Litjens, Premature aging of circulating $\mathrm{T}$ cells in patients with end-stage renal disease, Kidney Int. 80 (2) (2011) 208-217.

[31] R.W. Meijers, N.H. Litjens, D.A. Hesselink, A.W. Langerak, C.C. Baan, M.G. Betjes, Primary cytomegalovirus infection significantly impacts circulating T cells in kidney transplant recipients, Am. J. Transplant. 15 (12) (2015) 3143-3156.

[32] R.A. Montgomery, A. Loupy, D.L. Segev, Antibody-mediated rejection: new approaches in prevention and management, Am. J. Transplant. 18 (Suppl. 3) (2018) 3-17.

[33] T. Hirohashi, C.M. Chase, P. Della Pelle, et al., A novel pathway of chronic allograft rejection mediated by NK cells and alloantibody, Am. J. Transplant. 12 (2) (2012) 313-321.

[34] G. Benichou, Y. Yamada, A. Aoyama, J.C. Madsen, Natural killer cells in rejection and tolerance of solid organ allografts, Curr. Opin. Organ Transplant. 16 (1) (2011) 47-53.

[35] M.D. Parkes, P.F. Halloran, L.G. Hidalgo, Evidence for CD16a-mediated NK cell stimulation in antibody-mediated kidney transplant rejection, Transplantation. 101 (4) (2017) e102-e111.

[36] M. Crespo, J. Yelamos, D. Redondo, et al., Circulating NK-cell subsets in renal allograft recipients with anti-HLA donor-specific antibodies, Am. J. Transplant. 15 (3) (2015) 806-814.

[37] E. Suviolahti, S. Ge, C.C. Nast, et al., Genes associated with antibody-dependent cell activation are overexpressed in renal biopsies from patients with antibody-mediated rejection, Transpl. Immunol. 32 (1) (2015) 9-17.

[38] T. Legris, C. Picard, D. Todorova, et al., Antibody-dependent NK cell activation is associated with late kidney allograft dysfunction and the complement-independent alloreactive potential of donor-specific antibodies, Front. Immunol. 7 (2016) 288.

[39] J. Reeve, G. Einecke, M. Mengel, et al., Diagnosing rejection in renal transplants: a comparison of molecular- and histopathology-based approaches, Am. J. Transplant. 9 (8) (2009) 1802-1810.

[40] P.F. Halloran, J.M. Venner, K.S. Famulski, Comprehensive analysis of transcript changes associated with allograft rejection: combining universal and selective features, Am. J. Transplant. 17 (7) (2017) 1754-1769.

[41] G. Einecke, J. Reeve, M. Mengel, et al., Expression of B cell and immunoglobulin transcripts is a feature of inflammation in late allografts, Am. J. Transplant. 8 (7) (2008) 1434-1443

[42] M.G. Betjes, R.W. Meijers, E.A. de Wit, W. Weimar, N.H. Litjens, Terminally differentiated CD8 + Temra cells are associated with the risk for acute kidney allograf rejection, Transplantation. 94 (1) (2012) 63-69.

[43] B. Dedeoglu, R.W. Meijers, M. Klepper, et al., Loss of CD28 on peripheral T cells decreases the risk for early acute rejection after kidney transplantation, PLoS One 11 (3) (2016) e0150826. 